\title{
NUMERICAL EXPERIMENTS ON EXPANDING GRAVITATIONAL SYSTEMS
}

\author{
G. JANIN and M. J.HAGGERTY
}

European Space Operations Centre, Darmstadt, Germany, and The University of Texas at Austin, U.S.A.

It has been suggested that particle positions in expanding $N$-body gravitational systems may become increasingly correlated. Such incoherent density fluctuations could develop into bound clusters. Similarly superclusters of such clusters may be dynamically created. We present results of explorations of this idea, using computer simulation. Accurate integrations are made of gravitationally interacting systems of a few hundred particles.

The effect of the rest of the universe on the system is simulated by adding a smooth time-dependent gravitational field. The results are compared with the evolution of similar systems with more discrete particles placed outside. During the computer experiments a reduction of the number of bodies is sometimes made by reducing small subclusters to single particles at their centres of mass.

With near balancing kinetics and potential energies for the system, the rate of formation of well-isolated small clusters is lower than postulated for the simplest dynamical models of creation of hierarchical structures.

If the initial total potential energy slightly exceeds the initial expansion kinetic energy in magnitude, then our results suggest that a hierarchical density distribution is in the process of being created.

If the total energy is positive, then our results suggest a two-level hierarchy for the density distribution.

The correlations between binding energies, angular momenta and masses of small isolated clusters agree well with observations and with some previous theoretical and computational results. Indirect evidence is presented for the rapid production of massive but less conspicuous clusters.

A description of this study will be published in Astronomy and Astrophysics.

\section{DISCUSSION}

R. H. Miller: What do you mean by several orders of subclustering for initial energy $<0$ ?

G. Janin: Instead of speaking of several orders of clustering, one can introduce the less ambiguous notion of hierarchical clustering, meaning that the interaction between clusters or superclusters is similar to the interaction between particles in a cluster.

A. Poveda: If you have cases with negative initial energy, do you mean that after some time the initial expansion is reversed to a contraction of the whole system?

G. Janin: We have cases with slightly negative initial total energy. In these cases a part of the system keeps expanding.

D. C. Heggie: In the case when only one order of subclustering occurs, what is the distribution of the sizes of the subclusters? Do you get only pairs, or are there also triples, and so on?

G. Janin: In a typical case, if one defines the five following categories of clusters containing 1 star, 2-3, 4-7, 8-15, 17-31 stars, after two crossing times the proportion between the population of two successive categories is nearly $2: 1$. 